\title{
On the spectral distribution of kinetic energy in large-scale atmospheric flow
}

\author{
A. Wiin-Nielsen \\ Geophysical Deparment. Niels Bohr Institute for Astronomy. Physics and Geophysics, Juliane Maries Vej 30. 2100 Copenhagen. Denmark
}

Received: 27 April 1998 - Accepted: 14 October 1998

\begin{abstract}
A one-dimensional form of the equation of motion with forcing and dissipation is formulated in the spectral domain and used to make long term integrations from which the spectral distribution of the kinetic energy is determined. The forcing in the wave number domain is determined in advance and kept constant for the duration of the time integrations. The dissipation is proportional to the second derivative of the velocity.

The applied equation is made non-dimensional by selecting a length scale from which the time scale and the velocity scale may be determined. The resulting equation contains no parameters apart from the forcing. The integrations use a large number of spectral components and no approximation is made with respect to the non-lincar interaction among the spectral components. Starting from an initial state in which all the velocity components are set to zero the equation is integrated for a long time to see if it reaches a steady state.

The spectral distribution of the kinetic energy is determined in the steady state, and it is found that the distribution, in agreement with observational studies, may be approximated by a power law of the form $\mathrm{n}^{-3}$ within certain wave number regions. The wave numbers for which the -3 power law applies is found between the region of maximum forcing and the dissipation range.

The intensity of the maximum forcing is varied to see how the resulting steady state varies. In addition, the maximum number of spectral components is varied. However, the available computing power sets an upper limit to the number of components.
\end{abstract}

\section{Introduction}

The spectral distribution of the kinetic energy has been investigated in observational and model studies. The author (1967) found that the dependence on the longitudinal wave number for the eddy kinetic energy could be approximated by a power

Correspondence to: A. Wiin-Nielsen law of the form $\mathrm{n}^{-3}$ in a certain interval of wave numbers between the wave number of maximum conversion of eddy available potential energy to eddy kinetic energy and the smallest wave number in the dissipation range. A similar investigation by Horn and Bryson (1963) had suggested a power law with a coefficient closer to $-8 / 3$. The -3 power law was confirmed by independent data studies by Julian et al. (1970), Kao (1970) and Kao and Wendell (1970). An expansion of the observational studies to a spherical domain using a two-dimensional index was carried out by Chen and Wiin-Nielsen (1978).

Lilly (1969) made several numerical integrations of the two-dimensional, nondivergent vorticity equation with forcing and dissipation and found the -3 power law for wavenumbers larger than the wave numbers for the forcing. He found also a different -1 power law for wavenumbers smaller than the forcing wave numbers. The power laws have also been investigated in other model simulations as for example by Barros and Wiin-Nielsen (1974).

At about the same time as the first observational studies were published Kraichnan (1967) and Leith (1968) investigated the inertial or cascade ranges in two-dimensional flow. Leith (1971) used the results to determine the predictabilty of two-dimensional flows. Charney (1971) generalized the theory to the large-scale, three-dimensional, quasi-geotrophic flow in the atmosphere and gave thus a better reason for the observed -3 power law. Charney used a continuous vertical variation of the parameters, but Merilees and Warn (1972) pointed out that if one used a discontinuous representation given by a finite number of levels the results were modified, especially if the number of levels were at a minimum of just 2 levels.

The interest in turbulence studies created by the papers by Kraichnan and Leith (loc.sit.) continued by studies of a large number of so-called shell models. These models and their behavior are summarized by Ditlevsen (1995) and used for many purposes by Jensen (1992). Since it is impossible for practical reasons to include all the nonlinear interactions for the full three-dimensional equations of motion and at the same time include many spectral components, it has been 
attempted to reduce the interactions to those between a given wave number and its immediate neighbors in the spectral domain. At the same time it is attempted to include a very large range of wave numbers by dividing the total in shells in which the number of wave numbers in each shell is represented by a single wave number, and in which the waves numbers per shell increases by a factor of two when going from one shell to the next. These rather severe assumptions may limit realistic applications of such models, but some of the results are impressive.

In the present investigation we shall use a more direct approach by including as many spectral components as possible and make a full determination of all nonlinear interactions. This is made possible by restricting the problem to one dimension in space as was done in most observational studies where the wave number is the number of waves in the longitudinal direction. The other variations with latitude and height were averaged out in the data studies.

The basic equation applied in this study is Burgers equation with a forcing added to the classical equation. Burgers equation (Burgers, 1974, Fournier and Fritsch, 1983)) without forcing may be transformed to a lincar diffusion equation using a nonlinear transformation $\mathrm{u}=-2 v \varphi \%$ where the prime indicates differentiation with respect to $\mathrm{x}$, (Hopf, 1950). In view of the fact that the transformed equation is linear, solutions of the diffusion equation are easily produced. Some of these solutions are produced without forcing, while others include a forcing added to the diffusion equation. Applications cover many physical areas as described by Lesieur (1995).

In the present case we are interested in the spectral distribution of the kinetic energy when forcing is applied in Burgers equation. When the Hopf transformation is applied the forcing term will appear in a term that is the product of the integral of the forcing with respect to $x$ and the new variable $\varphi$. Due to this product the resulting equation must in general be solved using a spectral approach similar to the procedure described in the following sections. Nothing is therefore gained by making the transformation in this case, particularly because the transformation back to the original velocity field is also nonlinear.

\section{The basic equation.}

As mentioned in the introduction we use a one-dimensional equation. It has the form:

$$
\frac{\partial u}{\partial t}+u \frac{\partial u}{\partial x}=\frac{u_{E}}{T}+v \frac{\partial^{2} u}{\partial x^{2}}
$$

The term $u_{E} / T$ has been written in this form to secure that $u_{E}$ has the dimension of a velocily. ' $\mathrm{T}$ ' is the time scale. It will be determined below. We may also mention that this particular term is an expression for the gradient of a geopotential field $(-\delta \varphi / \delta x)$ which is constant in time. Eq. (2.1) may therefore also be considered as the equation for the velocity changes created by advection and by the influence of a geopotential field, constant in time, and modified by friction. To obtain the equation in the spectral domain we write the series given in (2.2). We could naturally include the cosine terms in the series, but we have prefered to keep the equations as simple as possible. If we prefer to think of the constant geopotential field it would have to be given as a sum of cosine terms.

Inserting the defined series in (2.1) it becomes necessary to pay special attention to the only nonlinear term: the advection term. It has been calculated by Wiin-Nielsen (1976).

$$
\begin{gathered}
u=\sum_{n=1}^{n m a x} u(n) \sin (n k x) \\
u_{E}=\sum_{n=1}^{n m a x} u_{E}(n) \sin (n k x) \\
k=2 \pi / L
\end{gathered}
$$

The spectral equation is given in (2.3), see also the appendix.

$$
\begin{gathered}
\frac{d u(n)}{d t}=S_{1}(n)-S_{2}(n)+\frac{u_{E}(n)}{T}-v k^{2} n^{2} u(n) \\
S_{1}(n)-1 / 2 k \sum_{n=1}^{n m a \cdot x-n} n u(q) u(n+q) \\
S_{2}(n)=1 / 2 k \sum_{n=1}^{n-1} q u(q) u(n-q)
\end{gathered}
$$

In the equation $k=2 \pi / L$, where $L$ is the length of the channel. The next step is make equation (2.3) nondimensional. For this purpose we replace the variables by new nondimensional variables as given in (2.4).

$$
u(n) \rightarrow U u(n) ; u_{E} \rightarrow U u_{E}(n) ; t \rightarrow T t
$$

Note that we use the same notation for the dimensional and the nondimensional quantities. Making the replacements in (2.3) we find that the coefficients become unity if we select the values given in (2.5). The final equation is given in (2.6). 


$$
T=\frac{1}{v k^{2}} ; U=2 v k
$$

$$
\begin{gathered}
\frac{d u(n)}{d t}-S_{1}(n)-S_{2}(n)+u_{E}(n)-n^{2} u(n) \\
S_{1}(n)=\sum_{q=1}^{n n n a x} n \\
S_{2}(n)=\sum_{q=1}^{n-1} q u(q) u(q+n)
\end{gathered}
$$

It is seen from eq. (2.1) that the mean value of $u$ is conserved over the interval $[0: \mathrm{L}]$ when $\mathrm{u}$ is either periodic over the interval or $\mathrm{u}$ vanishes at both ends of it. The same holds for eq. (2.6) because each term in the two sums is cancelled by an identical term with the opposite sign. For details of the cancellations see Wiin-Nielsen (1986). These conservation laws are important for any comparison with shell models in which conservation laws are used to determine the coefficients in the equations, see for example Wiin-Nielsen (1997) in which a shell model is designed using the conservations of the mean value and the kinetic energy. Another example may be found in Ditlevsen and Mogensen (1996) in which a shell model is designed using the conservations of kinetic energy and enstrophy.

\section{Numerical integrations of the model.}

The model requires a specification in advance of the forcing $u_{\bar{E}}[n]$. It can be done in many ways. We shall mostly use a definition in which $u_{E}[n]$ has a maximum for $n=n_{s}$ simulating the scale of maximum conversion of eddy available energy to eddy kinetic energy. It is known from observational studies that this maximum is found in the region of maximum baroclinic instability, say $n_{s}=7$, corresponding a wavelength of about $4000 \mathrm{~km}$ in middle latitudes.

It is for numerical reasons impossible to cover all wavelengths from the largest to the smallest in an integration of eq. (2.6). As the largest wavelength we may select $\mathrm{L}=$ $10000 \mathrm{~km}$. Such a wavelength is for normal vertical wind shears in the region of weak baroclinic instability. Suppose that we wanted to cover all scales down to $10 \mathrm{~km}$. In that case the largest wave number would have to be 1000 . Such an integration is very difficult to complete because it will take an extremely long time. We shall rather adopt the same point of view as used with three-dimensional prediction and climate models which are restricted to the larger scales. For $n_{\max }=100$ the smallest wavelength will be $100 \mathrm{~km}$. Such an integration may be performed in a reasonable time. Most integrations to be described will use the above or smaller values for the largest $n$. The main point is that the largest value of $n$ should permit a sufficient interval between $n_{s}$ and $n_{\max }$ in which we would hope to find an inertial range.

The integrations of the ordinary differential equations is carried out using the Heun scheme. Considering the scaling to non-dimensional equations it was found necessary to use a non-dimensional time-step of the order of $10^{-5}$. Such a value may be found by using the scaling value of $\mathrm{T}$ and $\mathrm{L}$ together with a value of $v=10^{5} \mathrm{~m}^{2} \mathrm{~s}^{-1}$. Although the time step is small it is only necessary to integrate to relatively small values for $\mathrm{t}_{\max }$ defined as the time in which the integration has reached a steady state. Experience shows that the steady state is reached for a value of $t_{\text {max }}$ of the order of 2 nondimensional units.

In the first example we have used $n_{\max }=50$ and $n_{s}=7$. The forcing has a maximum at $\mathrm{n}_{\mathrm{s}}=7$ and has been obtained from the following formulas:

$$
\begin{gathered}
1 \leq n \leq n_{s}: u_{E}[n]=u_{\operatorname{mix}} \frac{n}{n_{s}}\left(2-\frac{n}{n_{s}}\right) \\
n_{s} \leq n \leq n_{\max }: u_{E}[n]=u_{\max } \\
\left(1-\left(\frac{n-n_{s}}{n_{\max }-n_{s}}\right)^{2}\right)
\end{gathered}
$$

It is seen that $u_{E}[n]$ is zero for $n=0$ and equal to $u_{\text {ruax }}$ for $n$ $=n_{s}$, while the second formula shows that $u\left[n_{\text {max }}\right]=0$. The value of $u_{\text {mix }}$ in the example is 10 .

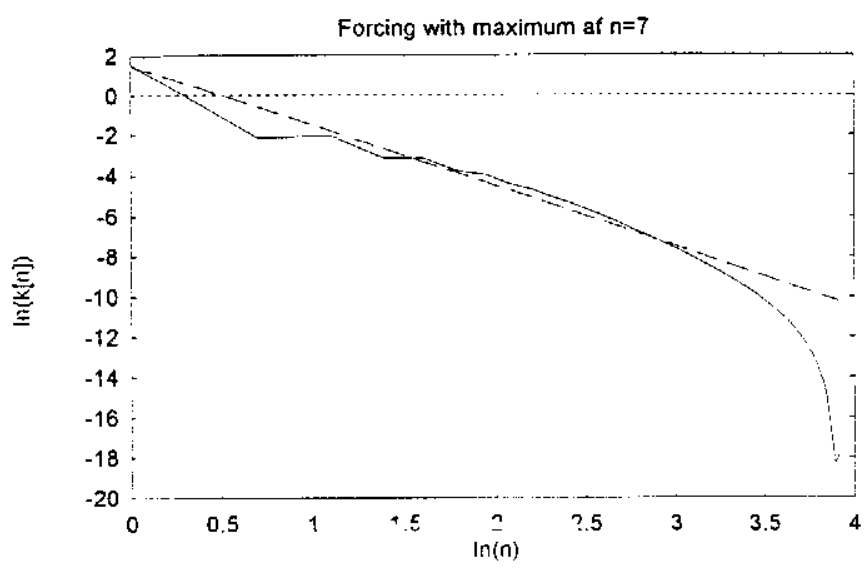

Fig. 1 The logarithm of the kinctic energy as a function of the logarithm of the wave number with a maximum forcing of $\mathrm{u}_{\mathrm{n}: \mathrm{iax}}=10$ at $\mathrm{n}=7$. The slope of the straight line is -3 . 
Fig. 1 shows the natural logarithm of the kinetic energy $k[n]$ as a function of the natural logarithm of $n$, when the integration with respect to time has been carried to a steady state starting initially from zero values of $\mathrm{u}[\mathrm{n}]$ for all $\mathrm{n}$. The total integration time is $\mathbf{t}=2$. The curve indicates a slight up and down variation for small values of $n$. Thereafter follows a curve that can be approximated by a straight line with the slope -3. For large values of $\ln (n)$ we see a clear indication of the dissipation term. To get an indication of the flux of the kinetic energy in the spectrum we have defined a fluxfunction. The definition is:

$$
\frac{\partial F}{\partial n}=-\left(S_{1}\left[n\left|-S_{2}\right| n \mid\right) u \mid n\right]
$$

from which $\mathrm{F}[\mathrm{n}]$ can be obtained by numerical integration. The fluxfunction is given in Fig. 2 for the steady state. It shows that the flux is from lower to higher values of $n$ for moderately large values of the wave number. For large values of $n$ the flux goes to zero. For small values of $n$ the flux goes in the opposite direction indicating a flux from larger to smaller values of the wave number. Fig. 3 shows the values of the two nonlinear sums and the difference between them. The two curves with positive values are $S_{1}[n]$ with the smaller positive values and $S_{2}[n]$ with the larger positive values giving a negative value of the difference as indicated by the third curve in the diagram.

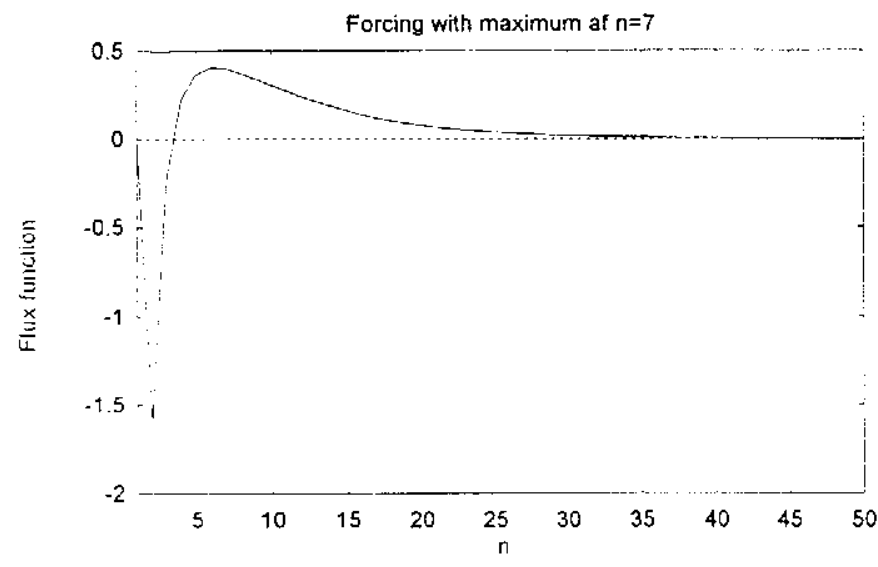

ligg. 2 The flux-finction for the case considered in Fig. 1 as a function of the wase number

The maximum forcing in the first case was $10 \mathrm{~m}$ per $\mathrm{s}$. In the second case we increase the maximum forcing to $20 \mathrm{~m}$ per $\checkmark$ while maintaining the maximum wave number at $\mathrm{n}_{\max }=50$. $\mathrm{F} 1 \mathrm{y} .4$ shows the kinetic energy as a function of wave number in the steady state. The -3 power law is found again, but in this ciase we are, in agreement with Lilly (1969), able to fit a priver law for the wave numbers smaller than $n_{s}$. The two straight lines cross very close to the wave number of maximum forcing $\left(\mathrm{n}_{\mathrm{s}}=7, \ln (7)=1.95\right)$.

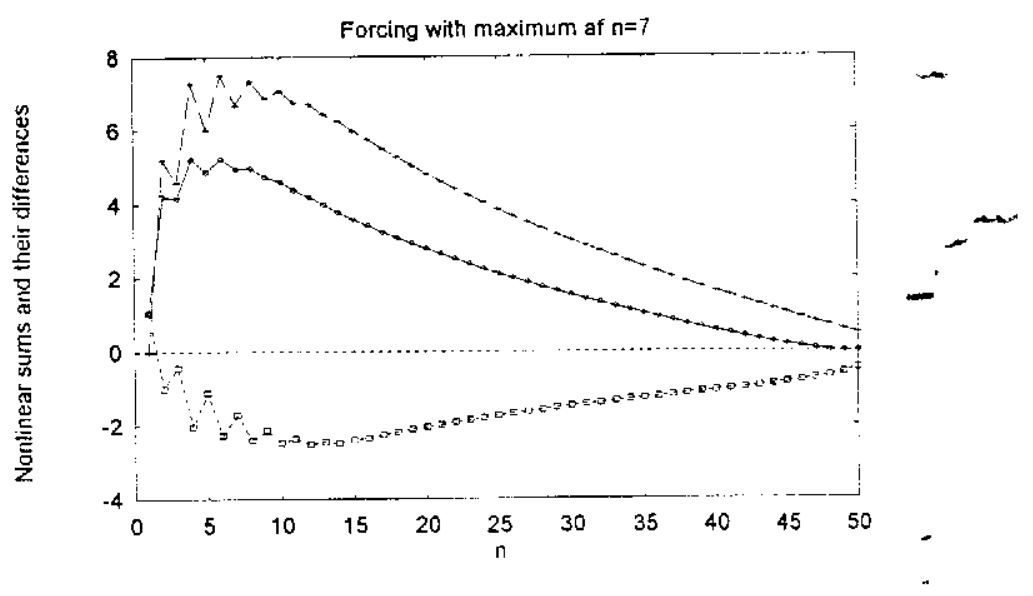

Fig. 3 The nonlinear sums entering the hasic equation and the difference of these sums. Note that $S_{l}[1]$ is the curve with the smaller positive values, while $S_{2}[n]$ has the large positive valucs giving a negative value for most wave numbers for the difference.

Integrations have also been carried out for various values of the maximum forcing and for a total number of wave components of 100 and 150 . The results are in agreement with those displayed in Fig. 4.

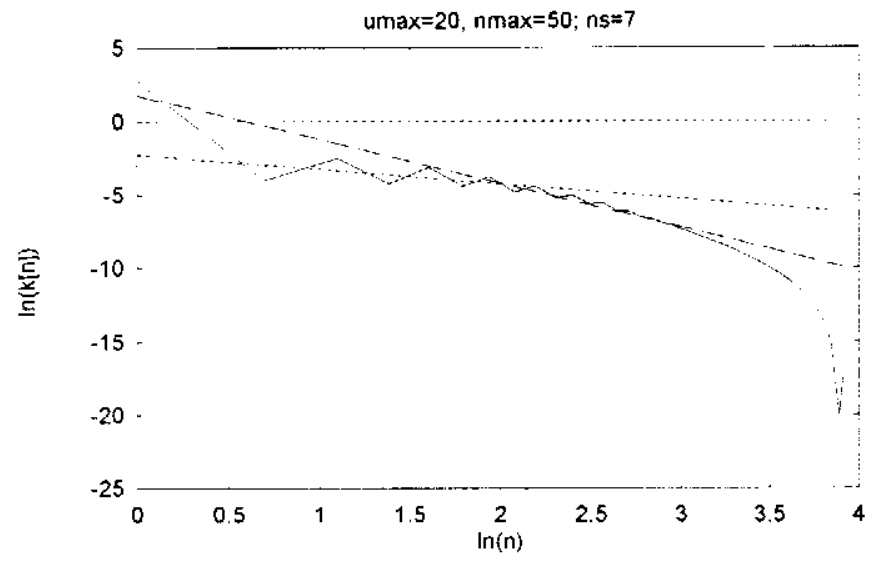

Fig. 4 The logarithm of the kinctic encrgy as a function of the logarithm of the wave number with a maximum forcing of $\mathrm{u}_{\text {anax }}=20$ at $\mathrm{n}=7$. The slope of the straight lines are -1 and -3 , respectively.

\section{Conclusions.}

For a sufficiently large value of the maximum forcing $\left(u_{111 x}=20\right)$ the experiments show a -3 power law in the wave number interval between the maximum forcing and the dissipation range (se Fig. 1 and 4 ). In addition a -1 power law can be detcrmined for wave numbers smaller than the wave 
number for maximum forcing. These results are obtained with a one-dimensional model based on the first equation of motion. The flux of kinetic energy is from smaller to higher wave numbers in the -3 range, while the flux is from larger to smaller wave numbers for the smallest wave numbers.

These results are at variance with results obtained from shell models. The author (1997) has designed a onedimensional shell model with the same conservation laws as in the full spectral model used in the present model, i.e. the conservation of the mean value and the conservation of the kinetic energy. However, the shell model gives a good approximation to the $-5 / 3$ power law. In addition, a shell model designed by Ditlevsen and Mogensen (1996) stressed the conservation of the kinetic energy and of enstrophy as in quasi-nondivergent large-scale flow, and they obtained a -3 law. It would thus appear that the results obtained with the various shell models are different from those obtained from a direct integration including all the nonlinear interactions.

\section{Appendix}

The purpose of this appendix is to give a derivation of the two sums appearing in eq. (2.3). The detailed derivation has not appeared before, but it has been used in a number of studies, Wiin-Nielsen (1976) and (Wiin-Nielsen, 1986). These reports are, however, not easily available. It is sufficient to consider the basic equation without the forcing and the dissipation, i.e.

$$
\frac{\partial u}{\partial t}+u \frac{\partial u}{\partial x}=0
$$

As mentioned earlier the variable $u$ is written as the series:

$$
u=\sum_{n=1}^{n m a x} u(n) \sin (n k x) ; k=2 \pi / L
$$

Inserting the series in (A.2) in (A.1) and using the three counters $n, r$ and $q$ we may write the result in the form:

$$
\frac{d u(n)}{d t^{\circ}}=-2 \sum_{r=1}^{n n n x} \sum_{q=1}^{n m a x}(q k u(r) u(q) I)
$$

where I is the interaction integral given by

$$
I=\frac{1}{L} \int_{0}^{L} \sin (r k x) \cos (q k x) \sin (n k x) d x
$$

Using elementary trigonometric formulas for the sums and differences of sine and cosines the integral I may be evaluated with the result that $\mathrm{I}=1 / 4$ when $\mathrm{r}=\mathrm{n}-\mathrm{q}, \mathrm{I}=1 / 4$ when $\mathrm{r}=\mathrm{n}+\mathrm{q}$ and $I=-1 / 4$ when $r=q-n$. Otherwise the interaction integral is zero. We find therefore a spectral equation as given in (A.4).

$$
\begin{gathered}
\frac{d u(n)}{d t}=1 / 2 \sum_{q=n+1}^{n \max } q k u(q-n) u(q) \\
-1 / 2 \sum_{q=1}^{n m \pi x-n} q k u(n+q) u(q) \\
-1 / 2 \sum_{q=1}^{n-1} q k u(n-q) u(q)
\end{gathered}
$$

The formula (A.4) could be used as it stands. A simplification can, however, be obtained by noting that when a new counter $q^{\prime}=q-n$ is introduced in the first sum it is transformed into the same summation as in the second term. These two terms may therefore be combined to a single term.

$$
\begin{gathered}
\frac{d u(n)}{d t}=1 / 2 n k \sum_{q=1}^{n \max -n} u(q) U(n+q) \\
-1 / 2 k \sum_{q=1}^{n-1} q u(q) u(n-q)
\end{gathered}
$$

The final equation is therefore as given in (A.5)

which is agreement with the sums as given in (2.3).

\section{Acknowledgement.}

The author should like to thank Dr. Peter D. Ditlevsen for very useful discussions on the content of this paper.

\section{References}

Burgers, J.M., 1974: The nonlinear diffusion equation, Reidel, Dordrechl. Charney, J.G., 1971: Geostrophic turbulence, J. Atmos. Sci., 28, 1087-1095. Ditlevsen, P.D., 1995: A shell model of cascade in tubulent flow, Modern Dynamical Mctcorology, Proc. of Symposium, Dept. of Geophysics, Univ. of Copenhagen, 163-168.

Ditlevsen, P.D. and I.A. Mogensen, 1996: Cascades and statistical equilitrium in sheli models of turbulence, Phys. Rev. E, 53 (5), 4785. Fjørtoft, R., 1953: On the changes in the spectral distribution of kinetic energy for two-dimensional nondivergent flow, Tellus, 5, 225-230.

Fournier, J.D. and U. Frisch, 1983: L'equation de Burgers déterminite et statistique, J.Mec.Theor.Appl., 2, 699-750

Hopf, E., I950: The partial differential equation $u_{s}+u u_{x}=\mu u_{x x}$, Commun. Pure and Appl. Math., 3, 201-230.

Horn, L.H. and R.A. Bryson, 1963: An analysis of the geostrophic kinetic 
energy spectrum of large-scalc atmospheric turbulcnce, Jour. of Gcophys. Res.,68, 1059-1064.

Jensen, M.H., G. Paladin, and A. Vulpiani, 1992: A shell model for turbulent advection of passive scalar fields, Phys. Rev. A, 45, 7214.

Jensen, M.H., 1994: Multifractals and mulitiscaling, D.Sc. diss., Niels Bohr Institute, Univ. of Copenhagen, $179 \mathrm{pp}$.

Julian, P.R., W.M. Washington, L. Hembrec and C. Ridlcy, 1970: On the spectral distribution of large-scale atmospheric kinetic energy, J. Atmos. Sci.,27,376-387.

Kao, S.-K., 1970: Wave number-frequency analysis spectra of temperature in the free atmosphere, J. Atmos. Sci,27,1000-1007.

Kao, S.-K. and L.L. Wendell, 1970: The kinetic energy of the large-scale atmospheric motion in wave number, frequency space: Northern Hemisphere, J. Atmos. Sci., 27, 359-375.

Kraichnan, R., 1967: Inertial Ranges in two-dimensional turbulence, Phys. Fluid, 10, 1417-1423.

Leith, C..E., 1968: Diffusion approximation for two-dimensional turbulence, Phys. Fluid, 11,671-672.
Leith, C.E., 1971: Atmospheric predictability and two-dimensional turbulence, J. Atmos. Sci.,28, 145-161.

Lesieur, M., 1995: Turbulence in tluids, Kluwer Acad. Publ., 514 pp.

Lilly, D.K., 1969: Numerical simulation of two-dimensional turbulcnec. Phys.Fluid, Suppl. II, 240-249.

Lilly, D.K., 1971: Numerical simulations of developing and decaying twodimensional turbulence, $J$ of Fluid Mech., 45, Part 2, 395-415.

Merilees,P.E. and T. Wam, 1972: The resolution implication of geostrophic turbulence, J. Atmos. Sci., 29, 990-991.

Wiin-Nielsen, A., 1967: On the annual variation and spcctral distribution of atmospheric energy, Tellus, 19, 540-559

Wiin-Nielsen, A., 1976: Predictability and climate variation illustrated by a low-order system, Proc of a Seminar on the Scientific Foundation of

Medium-Range Weather Forecasts, ECMWF, 258-306.

Wiin-Nielsen, A., 1986: Studies of the advection equation with forcing and dissipation, Danish Meteorological Institute, Meddelelser, Nr. 28, 75 pp Wiin-Niclsen, A., 1997: On helicity and shell models, Dyn. of Oceans and Atmospheres, 27, 707-714. 